\title{
Determination of Uncertainty in Calibration of Acoustic Emission Sensors
}

\author{
Jiri Keprt \\ Department of Control and Instrumentation, Brno University of Technology, Czech Republic \\ keprt@feec.vutbr.cz \\ Petr Benes \\ Department of Control and Instrumentation, Brno University of Technology, Czech Republic \\ benesp@,feec.vutbr.cz
}

\begin{abstract}
The paper reviews the background, the methodology and the standardization of the primary calibration of acoustic emission sensors. There are discussed the aims and the purpose of the primary calibration. Two basic calibration methods, which were practically realized on our test stand, are described and explained. It is the method of reciprocity calibration and the step function calibration. As a transfer medium for wave propagation a large steel block was used.

The comparison of the results of the both method is presented. There shape of calibration characteristics corresponds well, but there was a difference in amplitude. Uncertainty of measurement by reciprocity calibration is determined and presented in the paper. The uncertainty in range from $60 \mathrm{kHz}$ to $285 \mathrm{kHz}$ was $\pm 3 \mathrm{~dB}$ and from $285 \mathrm{kHz}$ to $1 \mathrm{MHz}$ up to $\pm 8 \mathrm{~dB}$. Also the main problems, that were solved and have to be solved, are presented.
\end{abstract}

Keywords: Acoustic Emission, Sensor Calibration, Reciprocity Calibration, Step Function Calibration, Uncertainty.

\section{Introduction}

Acoustic Emission (AE) is a passive non-destructive testing technique that has been used widely since the 1970s. AE has been defined as the spontaneous release of elastic energy by material when it undergoes deformation.

Good metrology of the AE calibration method is necessary to be able to compare the results of calibration made by other laboratories or to compare the effects of ageing, thermal cycling and so on. ASTM E1106 [1] outlines a method for primary calibration of AE sensors and ASTM E976 [5] and E1781 [4] for secondary calibration.

The basic problems of calibration are [8]:

- The instantaneous displacement of a point at the surface is a three-dimensional vector but the output of the transducer is a scalar quantity.
- The loading effect due to the mechanical impedance of the transducer on the surface of the block affects measurement.

- Output signal is a function of the displacement of the measured surface in contact with the sensor. The function is not only dependent on time but also on the position within the measurement area of the transducer.

From previous investigations follow some assumptions, which it is necessary to take into account. The first condition is that transducers used for acoustic emission measurement are in general sensitive only to motion normal to the surface to which they are attached. Secondly, it is assumed that the loading effect due to the mechanical impedance of the transducer on the block surface has no effect on the displacement of the surface under the transducer. Nevertheless it is important to note the material of the testing block used in reporting the result.

The finite size of the sensor surface is often missed out. The sensor surface covers certain area on the surface on testing block. The displacement of the block surface under the sensor is not constant. The sensor responds to weighted average of the displacement. This effect is called aperture effect. The output voltage of the sensor is described as follow [10]:

$$
U(t)=\frac{1}{A} \iint_{S} u(x, y, t) r(x, y) d y d x
$$

where $r(x, y)$ is the local sensitivity of the transducer face, $S$ is the region $\left[\mathrm{m}^{2}\right]$ of the surface contacted by the transducer, $A$ is the area $\left[\mathrm{m}^{2}\right]$ of region $S$ and $u(x, y, t)$ is the displacement $[\mathrm{m}]$ on the surface.

For a sensor having a circular aperture (mounting face) with uniform sensitivity over the face, there are frequencies at which nulls in the frequency response occur. These nulls occur at zeroes of the first order Bessel function [1]

$$
J_{1}(k a)=0
$$

where $k=2 \pi f / c\left[\mathrm{~m}^{-1}\right], f$ - frequency [Hz], $c$ - the Rayleigh speed in the test block $\left[\mathrm{m} . \mathrm{s}^{-1}\right], a-$ the radius of the sensor face $[\mathrm{m}]$. Therefore, the calibration results 
depend on the Rayleigh wave speed in the material for the test block [1].

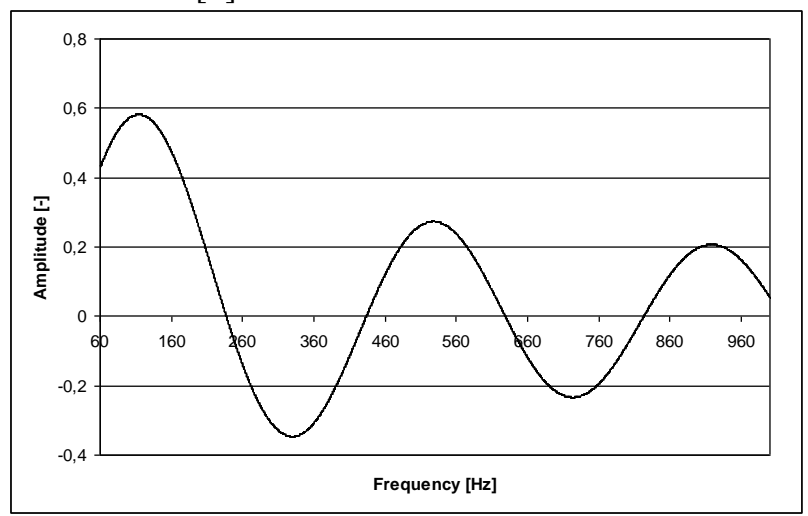

Figure 1: Calculated aperture effect for UT 1000

On the Figure 1, there is a shape of curve of calculated equation (2) for sensor UT 1000 with radius $0,775 \mathrm{~cm}$. Zero points are $237 \mathrm{kHz}, 433 \mathrm{kHz}, 628 \mathrm{kHz}$ and $823 \mathrm{kHz}$ for Rayleigh wave speed $3006 \mathrm{~m} . \mathrm{s}^{-1}$ for our testing block.

We can ignore this effect if the radius of the active element is negligible compared with length of the wave. For example, a sensor with diameter $3 \mathrm{~mm}$ can be used up to a frequency of $500 \mathrm{kHz}$ in a steel block with an acceptable measurement error.

The coupling of the sensor to the structure can be a major source of poor repeatability. There are three main aspects: the structure preparation prior to mounting of the sensor, the selection of the adhesive, and the choice of the mounting fixture. Preparation of the structure is highly important, the surface quality or the presence of other layers, oxide or paint for example, can affect the acoustic emission signal greatly. On a clean surface it is then important to attach the sensor each time with a consistent pressure and an adhesive that will not degrade with time.

The temperature can affect the properties of an adhesive and the piezoelectric constant [10].

Most calibration systems use AE sources and sensors placed on the same surface and hence are called "surface calibration". Most of the energy comes from Rayleigh waves and the results of the calibration reflect the sensitivity of the sensor for Rayleigh surface waves. The aperture effect is considerable here. Some systems use a different configuration of sensors and source, the tested sensor is mounted on the opposite side to the source on the collinear surface. A spherical wave is received by the transducer, but its curvature is so negligible that it can be considered to be planar and the result of the calibration is not affected by the diameter of the active element. This is called "through calibration".

\section{AE sensors}

Transducers used for acoustic emission measurement are in general sensitive to motion normal to the surface to which they are attached. Typically, AE transducers are sensitive to frequencies above $100 \mathrm{kHz}$. Resonant transducers are highly sensitive to a very narrow frequency range, which must be carefully selected depending on the application. Resonant transducers in the range $150 \mathrm{kHz}$ to $300 \mathrm{kHz}$ are probably the most widely used in AE applications. The highest frequencies likely to be of interest to users of $\mathrm{AE}$ transducers are in the range from $800 \mathrm{kHz}$ to $1 \mathrm{MHz}$.

There are several ways how this transduction can be achieved. The piezoelectric effect, capacitance methods and optical interferometry are common techniques used for detection of $\mathrm{AE}$ in industry and research. Piezoelectric devices offer the greatest sensitivity and thus they are the most widely used type of transducer in acoustic emission applications. When broad bandwidth measurement is of primary importance then optical and capacitive techniques are employed. Interferometers and capacitance transducers have the capability to perform very high fidelity measurements over a broad frequency range far beyond that of conventional piezoelectric transducers, often over tens of MHz. Interferometers also provide very good spatial resolution, which is ideal when measurements of small changes in surface displacement are required. Both the latter techniques are, however, relatively insensitive, being orders of magnitude less sensitive than a typical AE piezoelectric sensor. Furthermore, these devices can be very delicate and are commonly limited to laboratory use where measurement conditions can be controlled. Interferometers and capacitance transducers are often used to calibrate piezoelectric AE transducers.

Tab 1: Typical sensitivities of AE sensors [9]

\begin{tabular}{|l|l|l|}
\hline $\begin{array}{l}\text { TYPE OF } \\
\text { TRANSDUCER }\end{array}$ & $\begin{array}{l}\text { SENS } \\
\text {. [m] }\end{array}$ & $\begin{array}{l}\text { BANDWIDTH } \\
\text { [MHz }]\end{array}$ \\
\hline Piezoelectric resonant & $10^{-13}$ & 0,1 to 0,3 \\
\hline $\begin{array}{l}\text { Piezoelectric } \\
\text { wideband }\end{array}$ & $10^{-12}$ & 0,1 to 2 \\
\hline Capacitance & $10^{-11}$ & DC to 50 \\
\hline Laser interferometer & $10^{-10}$ & 0,05 to 100 \\
\hline
\end{tabular}

The most widely used type of a transducer in acoustic emission testing is a piezoelectric device.

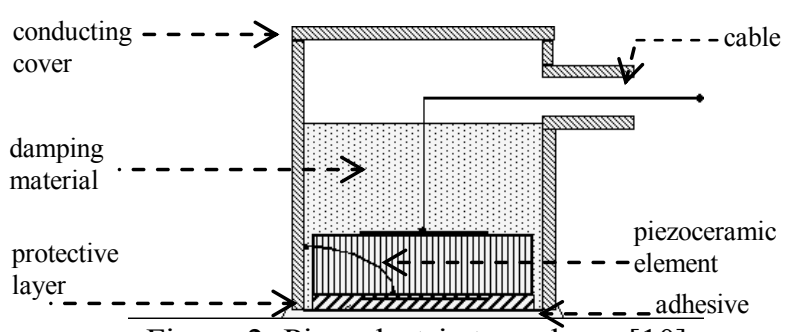

Figure 2: Piezoelectric transducer [10]

\section{AE sensors calibration}

A main problem of the calibration is to find the characteristic of the transducer. A frequency response of a specific sensor in the mechanical input quantity (velocity, displacement) is the most common result of calibration. Output quantity of calibration is output stress relate to unit of mechanical input (displacement, velocity, acceleration). The absolute value of input quantity and its 
shape has to be known for primary calibration. Each method has its specific limitations [19].

There are two types of AE sensor calibration:

For primary calibration the absolute value of input signal and its shape has to be known. A mechanical source of input signal with standard characteristics (shape of waveform - step or impulse, duration) is important for this. The method of calibration with a step function is described in [1] and reciprocity calibration in [2].

Secondary calibration requires a reference sensor with known characteristics. Calibration is done by comparison of the results of the reference and tested transducers. Data from the secondary calibration is of the same type as from the primary, but is more limited (in frequency, absence of shift characteristics, and greater error of calibration)[4].

\subsection{Step function calibration}

The basis for the step function force calibration is that known, well characterized displacements can be generated on a plane surface of a test block. A step function force applied to a point on one surface of the test block initiates an elastic disturbance that travels through the block. In general it is possible to use configuration of sensor for surface calibration and for through-pulse calibration. But through pulse calibration is use only for additional measurements.

It uses a standard reference capacitance transducer and the step-force is generated by the fracture of a glass capillary.

The response of the sensor being calibrated to the stepforce source is then compared with the reference transducer, which measures the surface displacement due to the elastic surface waves. The displacement at the reference transducer can also be calculated using elastic theory. The surface motion on the transfer block, determined using either technique, is the free motion of the surface and not the loaded surface displacement, under the transducer being calibrated. The loading effect of the sensor being calibrated, therefore, affects the measurement being made and thus becomes part of the calibration.

The measured data are used to calculate a fast Fourier transform to determine values of the spectra from unknown and reference sensor. The response of the transducer under test is as follows

$$
D\left(f_{m}\right)=\frac{U\left(f_{m}\right)}{S\left(f_{m}\right)}
$$

where $U\left(f_{m}\right)$ is spectrum from unknown sensor and $U\left(f_{m}\right)$ is spectrum from standard sensor or from solution of elastic theory.

The calibration is described in ASTM standard 1106[1]. The National Institute of Standards and Technology use follow calibration facility: a cylindrical steel test block $0.9 \mathrm{~m}$ in diameter $0.43 \mathrm{~m}$ long with optically polished end faces [10].
It is generally assumed that a transducer has only normal sensitivity because of its axial symmetry (an assumption that may not be justified). Calibration by the surface pulse technique for a transducer having significant sensitivity to tangential displacement will be in error, because the surface pulse from the step force contains a tangential component approximately as large as the normal component [10].

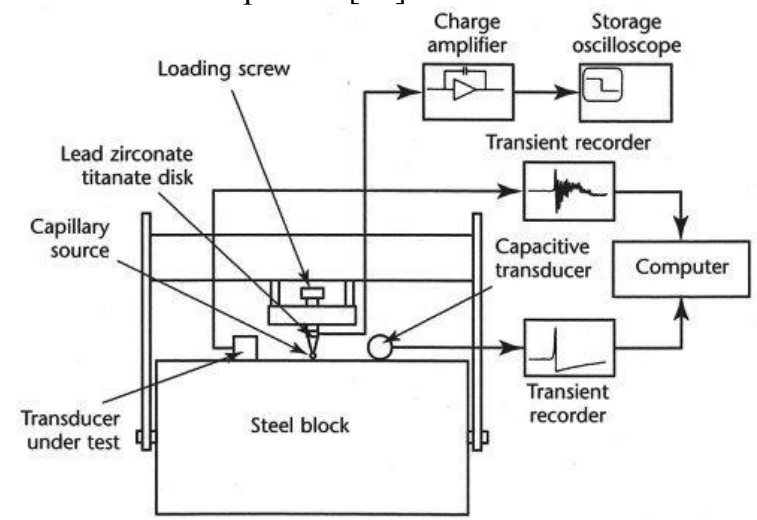

Figure 3: Schematic diagram of the step function calibration apparatus [10]

\subsection{Reciprocity calibration}

Reciprocity calibration works on reciprocity theorem that is known from electrical circuits:

"A voltage source $U$ acting in one branch of a network causes a current I to flow in another branch of the network, then the same voltage source $U$ acting in the second branch would cause an identical current I to flow in the first branch."

This principal can be use for electromechanical system and makes relation between transition of the sensor acting as source and later as receiver.

Reciprocity applies to a category of passive electromechanical transducers that have two important characteristics according to [10]:

1. They are purely electrostatic or purely electromagnetic in nature.

2. They are reversible - can be used as either a source or a receiver of mechanical energy.

This category includes all known commercial acoustic emission transducer without preamplifiers [10]. Sensor with preamplifiers can be calibrated by modification of this method by Adams [20].

With nonidentical transducers three measurements using each of three possible pairs (Figure 4) of transducer must be measured to obtain enough information to determine all of the response functions of the transducers absolutely.

The input current and reception signal voltage for tone bursts of varying frequency are established for each pair together with the reciprocity parameter, allowing each transducer to be calibrated by measuring electrical signals only. The transducer characteristics are defined as the transmission voltage response in the transmitter configuration and the free-field voltage sensitivity in the receiver configuration. 
The primary advantage of the reciprocity calibration technique is that it avoids the necessity of measuring or producing a known mechanical displacement or force. All of the basic measurements made during the calibration are electrical. It is important to note, that the mechanical transfer function or Green function for the transmission of signals from the source location to receiver location must be known. This function is equivalent to the reciprocity parameter, that describes a transfer function of a Rayleigh-wave and it takes into account the frequency of the Rayleigh wave and the material properties of the propagating medium. It is the frequency domain representation of the elasticity theory solution [10].

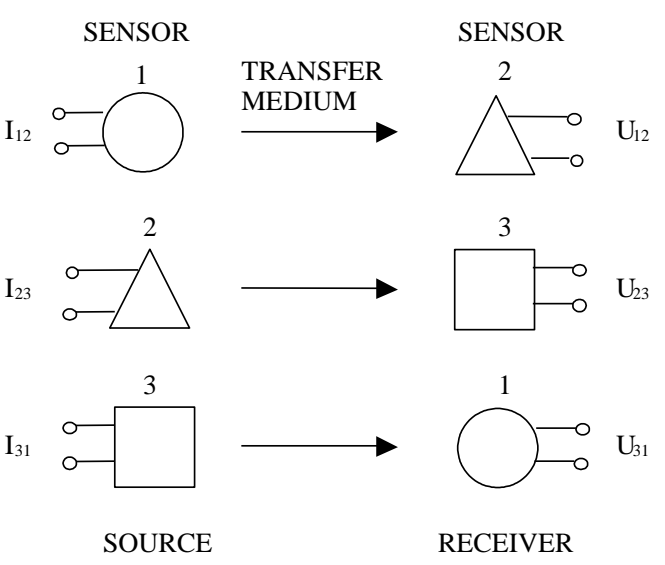

Figure 4: Reciprocity calibration for reversible transducers [6]

\section{Investigation of reciprocity calibration at FEEC BUT}

The selection of instruments was limited by the capability of the laboratory and by instruments used by Hatano at al. [6]. Here is the list of components used:

- Vector signal analyzer HP 89410A, two channels from DC up to $10 \mathrm{MHz}, \mathrm{GPIB}$ interface, sampling rate up to $25,6 \mathrm{MHz}$

- Arbitrary waveform generator HP 33220A. A waveform can have up to 64000 points, resolution 14 bits, $40 \mathrm{MSa} / \mathrm{s}$, GPIB interface

- Current probe Tektronix P6022 with impedance matching. Sensitivity $1 \mathrm{~mA} / \mathrm{mV}$ or $10 \mathrm{~mA} / \mathrm{mV}$. Bandwidth on range $1 \mathrm{~mA} / \mathrm{mV}$ is $8,5 \mathrm{kHz}$ up to $100 \mathrm{MHz}$

- $\quad$ Personal Computer Pentium 4, 3,2 GHz, 1024 MB RAM, GPIB-USB, OS Windows XP

- $\quad \mathrm{AE}$ amplifier with gain $30 \mathrm{~dB}$ in range from 35 $\mathrm{kHz}$ to $4 \mathrm{MHz}$ for drop $3 \mathrm{~dB}$

- Steel cylinder with diameter $94,0 \mathrm{~cm}$, height $43,8 \mathrm{~cm}$. Used material is signed $34 \mathrm{MnV}(0,34$ C). To suppressed interference the block was grounded with HP 89410A

- Adhesive paste Krautkramer TGT, number 50472

- $\quad$ GPIB and sensor cables

The duration of the driving signal was $100 \mu \mathrm{s}$ according to the size of the testing block. The first reflected wave from the bottom surface came after 150 $\mu \mathrm{s}$. The sampling rate was $25,6 \mathrm{MHz}$. The source signal was generated from 8000 points. The experiment was controlled by software developed in LabVIEW 8.2. The frequency transmission was probed in the range from 60 $\mathrm{kHz}$ to $1 \mathrm{MHz}$ point to point with step size $5 \mathrm{kHz}$. The resulting characteristic has only 189 frequency points and the experiment took 1,2 hour. The low frequency is limited by the duration of the input signal and the high frequency is limited by the sensitivity of the transducer.

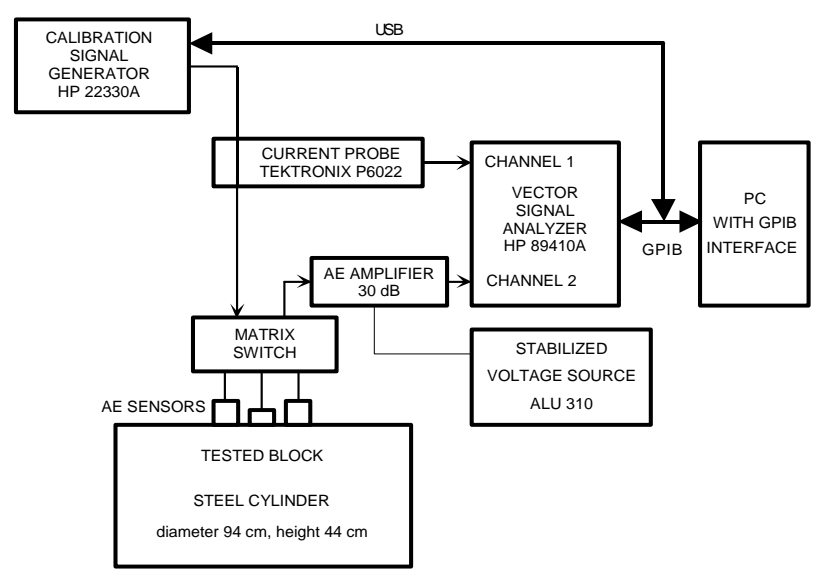

Figure 5: Schema of test rig configuration

\section{Investigation of step function calibration at FEEC BUT}

The driving signal for the step function is generated by break of glass capillary. A holding fixture was manufactured for it. The basis apparatus was used the same as for reciprocity calibration, but it was supplement according to ASTM E 1106 [1]:

- Force sensor LCMFD-100N. Range $\pm 100 \mathrm{~N}$, sensitivity $0,180067 \mathrm{mV} / \mathrm{N}$

- Vishay P - 3500 portable strain indicator with an analog output with a $-3 \mathrm{~dB}$ bandwidth of 4 $\mathrm{kHz}$

- Holding fixture for apparatus for capillary breaking

- Glass fibre with diameter smaller than 0,2 mm, manufactured from laboratory glass

- Laser interferometer Polytec OFV-5000 with optical head OFV-505 and computing module DD-300 for AE

Analyzer $89410 \mathrm{~A}$ was driven by trigger $0,05 \mathrm{~V}$ and $200 \mu \mathrm{s}$ of oncoming signal was sampled. Pretrigger $40 \mu \mathrm{s}$ was used and sampling frequency was $25,6 \mathrm{MHz}$. Signal of the capillary break was calculated by Matlab with the same length and sampling parameters as real signal. Signal generated from Matlab was recalculated from displacement to velocity and FFT was calculated from both signals. Final characteristic is calculated by the dividing measured spectrum by calculated spectrum (3). 
Standard deviations as characteristics of the spread of a random quantity are estimated by the experimental standard deviation $s\left(\bar{x}_{i}\right)$. Standard deviation $s^{2}\left(x_{i j}\right)(8)$ obtained from the definition of the variance.

$$
s^{2}\left(x_{i j}\right)=\frac{1}{n_{i}-1} \sum_{j=1}^{n_{i}}\left(x_{i j}-\bar{x}_{i}\right)^{2}
$$

The factor $n_{i}-1$ in the denominator instead of $n_{i}$ is due to fact that one degree of freedom is lost through the formation of the arithmetic mean.

The best estimate $s\left(\bar{x}_{i}\right)$ of the positive square root of the variance $\sigma^{2}\left(\bar{x}_{i}\right)=\frac{\sigma^{2}}{n_{i}}$ of the arithmetic mean $\bar{x}_{i}$ is then

$$
s\left(\bar{x}_{i}\right)=\frac{s\left(x_{i j}\right)}{\sqrt{n_{i}}}
$$

$s\left(\bar{x}_{i}\right) \ldots$ experimental standard deviation

$s\left(\bar{x}_{i}\right)$ was used as a uncertainty of type $\mathrm{A}$ for measurements of voltage and current.

\subsection{Uncertainty type B}

Main source of uncertainty of type B was vector signal analyzer HP 89410A. Its absolute amplitude full-scale accuracy is $\pm 0.5 \mathrm{~dB}$ from full-scale [16]. Converted to percent the error of the measurements can be up to $6 \%$ from input range. Provided that the error has homogenous rectangular distribution, the uncertainty of type $\mathrm{B}$ be is calculated as follow

$$
s(x)=\frac{0,06 * \text { Range }}{\sqrt{3}}
$$

\subsection{Combined uncertainty}

Because of simultaneous measurements of voltage and current for each pair it was assumed that at least two input quantities are interdependent. So the correlation for each combination of variables was calculated. Final uncertainty was calculated according

$$
\begin{array}{r}
u_{c}^{2}(y)=\sum_{i=1}^{N}\left(\frac{\partial f}{\partial x_{i}}\right)^{2} u^{2}\left(x_{i}\right)+2 \sum_{i=1}^{N-1} \sum_{j=i+1}^{N} \frac{\partial f}{\partial x_{i}^{(k)}} \frac{\partial f}{\partial x_{i}^{(k)}} u\left(x_{i}^{(k)}, x_{j}^{(k)}\right) \\
\frac{\partial f}{\partial x_{i}^{(k)}} \frac{\partial f}{\partial x_{i}^{(k)}} u\left(x_{i}^{(k)}, x_{j}^{(k)}\right)=u_{A}\left(x_{i}, x_{j}\right)
\end{array}
$$

\section{Evaluation of Covariance}

An estimate of the covariance of two correlated quantities was obtained using statistical methods. Because simultaneous series of measurement (voltage, current) were made, the arithmetic means of the series estimate the expectations of the two quantities. So the covariance was estimated according [15]

$$
u_{A}\left(x_{i}, x_{j}\right)=\frac{1}{n(n-1)} \sum_{k=1}^{n}\left(x_{i k}-\bar{x}_{i}\right)\left(x_{j k}-\bar{x}_{j}\right)
$$

The assumption that the variables are correlated was not confirmed and from calculations follows, that the calculated covariance was negligible.

\subsection{Expanded uncertainty}

In some application it has some merits to state an interval that, with a given probability, contains the value of the measurand. The interval and the value of the coverage factor were chosen in accordance with coverage probability at least $95 \%$. The value of coverage factor was 2.

\subsection{Results}

Uncertainty type A was calculated for all measured voltages and currents. Uncertainty of the $\mathrm{AC}$ current probe Textronix type A was calculated from the measured data of its characteristics.

Uncertainty type B was estimated from amplitude accuracy of signal vector analyzer HP 89410. It was calculated for all measured voltages and currents.

Than was calculated the combined uncertainty according (11) and finally the expanded uncertainty.

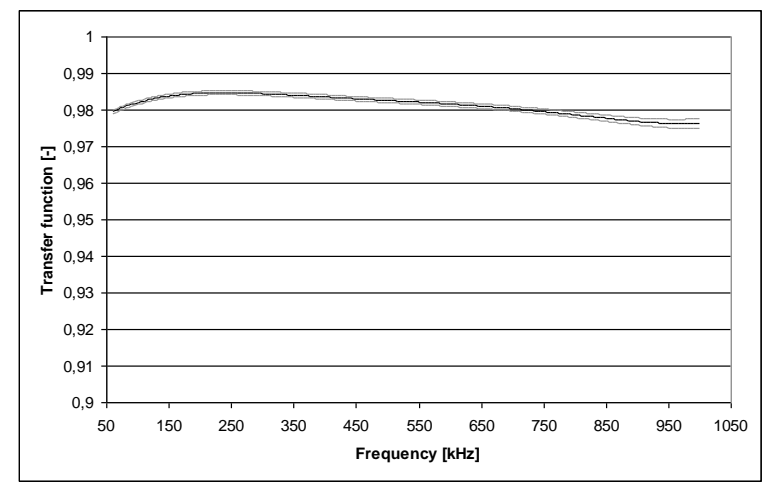

Figure 10 : Characteristic of current probe $\mathrm{P} 6022$

Characteristic of AC current probe Textronix P6022 (figure 10) was calculated from 10 measurement by mean. The voltage from current probe was compared to voltage on resistor with known value of resistance. The shape of excitation signal was the same, as used for excitation of AE sensor. All data measured by current probe was corrected by measured characteristics and the uncertainty of probe was included in combined uncertainty. 


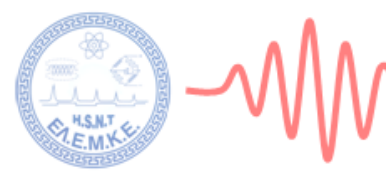

[14] Keprt, J. Reciprocity Methods of Acoustic Emission Sensor Calibration, Proceedings of The Annual Researchers Conference. University of Huddersfield, 2005, p. $63-66$

[15] Hruška, K. - Bradík, J., Stanoveni nejistot při měreni parametrů jakosti. VUT v Brně, 2001. 114 pages. ISBN 80-214-1656-1

[16] HP 89410A/HP 89441A Operator's Guide, HewlettPackard Company, 1998

[17] Laaneots, R., Mathiesen, O., An introduction to metrology, Estonia, 2006, ISBN 9985-59-609-9

[18] Palenčár, R., Vdoleček, F., Halaj, Nejistoty $v$ měrení III: Nejistoty nepřimých měrení, Automa 12, 2001, p. $28-33$

[19] Hsu, N. N., Breckenridge, F. R., Characterization and Calibration of Acoustic Emission Sensors, International Conference on Acoustic Emission, Anaheim, 1979, p. 60-69

[20] Hill, R., Adams, N. L., Reinterpretation of the Reciprocity Theorem for the Calibration of Acoustic Emission Transducers Operating on a Solid, Acustica, 1979, vol. 43, p. $305-312$.

\section{Acknowledgement}

This research has been supported by Grand Agency of the Czech Republic under the contract GACR 101/06/1689 and by the Czech Ministry for Education in frame of the Research Intention JC MSM 0021630529: Intelligent systems in automation. 